\title{
Multifunctional Composites Obtained by Incorporating Nanocrystals into Decorated PVK Polymers
}

\author{
Haizhu Sun, ${ }^{1,2}$ Junhu Zhang, ${ }^{1}$ Ye Tian, ${ }^{2}$ Yang Ning, ${ }^{1}$ Hao Zhang, ${ }^{1}$ Jie Ju, ${ }^{1}$ Delong Li, ${ }^{1}$ \\ Shidong Xiang, ${ }^{1}$ and Bai Yang ${ }^{1}$ \\ ${ }^{1}$ State Key Lab for Supramolecular Structure and Materials, College of Chemistry, Jilin University, Changchun 130012, China \\ ${ }^{2}$ College of Chemistry, Northeast Normal University, Changchun 130024, China
}

Received 28 March 2007; Accepted 4 October 2007

Recommended by Wieslaw Strek

Poly(vinylcarbazole) (PVK) was decorated with surfactant group to achieve amphiphilic polymer with luminescent property. The composition and properties of the polymers were systematically investigated using FTIR, EA, TGA, UV-Vis, and PL characterizations. Different CdTe nanocrystals (NCs) prepared in aqueous medium were directly transferred to organic phase using the PVK-based polymers. The quantum yield of NCs in the composites had been improved by $50 \%$ compared with their parent aqueous solution due to the short distance from carbazole moieties to NCs, which facilitated the Förster resonant energy transfer (FRET) between them. Moreover, efficient electron transfer at the interface of NCs and polymers had been confirmed which also indicated the application in photovoltaic cell for such composites.

Copyright (c) 2007 Haizhu Sun et al. This is an open access article distributed under the Creative Commons Attribution License, which permits unrestricted use, distribution, and reproduction in any medium, provided the original work is properly cited.

\section{INTRODUCTION}

Nowadays, a novel kind of luminescent polymers, namely, polymer with amphiphilic groups, has been extensively studied because such kind of polymers possesses several advantages. (1) They can offer particular properties compared with the normal neutral polymers due to their ionic side groups attached to the main chain. (2) Most amphiphilic polymers are typically soluble in water or alcohol, which are environment-friendly solvents. (3) Amphiphilic polymers are important building blocks for nanocrystal assembly via electrostatic interaction [1]. These advantages donate amphiphilic polymers with much more extensive applications; they can be used as the active layer in light-emitting diodes (LEDs) through layer-by-layer self-assembly method [2]; they are considered as highly sensitive materials in biological [3] and chemical sensors as well [4].

Semiconductor nanocrystals (NCs) directly synthesized in aqueous solution using water-soluble thio-compounds as ligands have intrigued much interests in recent years, because they are cheaper, less toxic, and biocompatible in comparison with the ones prepared in nonaqueous synthesis [5-8]. Many researchers pay attention to how to incorporate NCs into the polymers, because people expect that the resulted NC-polymer composites will combine the ad- vantages of both NCs and polymers. Luminescent polymers with amphiphilic groups naturally become one of the best choices to realize this aim. In our recent work $[9,10]$, we have developed a novel method to incorporate NCs into functional amphiphilic polymers. First, we designed a novel amphiphilic copolymer of poly(9-vinylcarbazoleco-octadecyl-4-vinylbenzyl-dimethyl-ammonium chloride) (CPVKOVDAC) with blue fluorescence. Then, NCs in aqueous phase were transferred to organic phase using the polymers to achieve NC-polymer multifunctional composites. Moreover, this method provided a chance of incorporating several different color-emitting NCs into a functional polymer. Therefore, by incorporating two types of CdTe NCs having two distinct PL emissions at 524 and $650 \mathrm{~nm}$ into the polymer, white-light PL and electroluminescence (EL) have been obtained from the solution processable NC-polymer composite. However, the quantum yield (QY) of the composites was low and hence we could not get the EL devices with high efficiency.

In this paper, we directly grafted surfactant group on poly(vinylcarbazole) (PVK) (see Scheme 1) in order to achieve PVK-based amphiphilic polymer. Different CdTe NCs prepared in aqueous medium were directly transferred to the organic phase using the PVK-based polymers. Compared with the CPVKOVDAC copolymer which we used in 

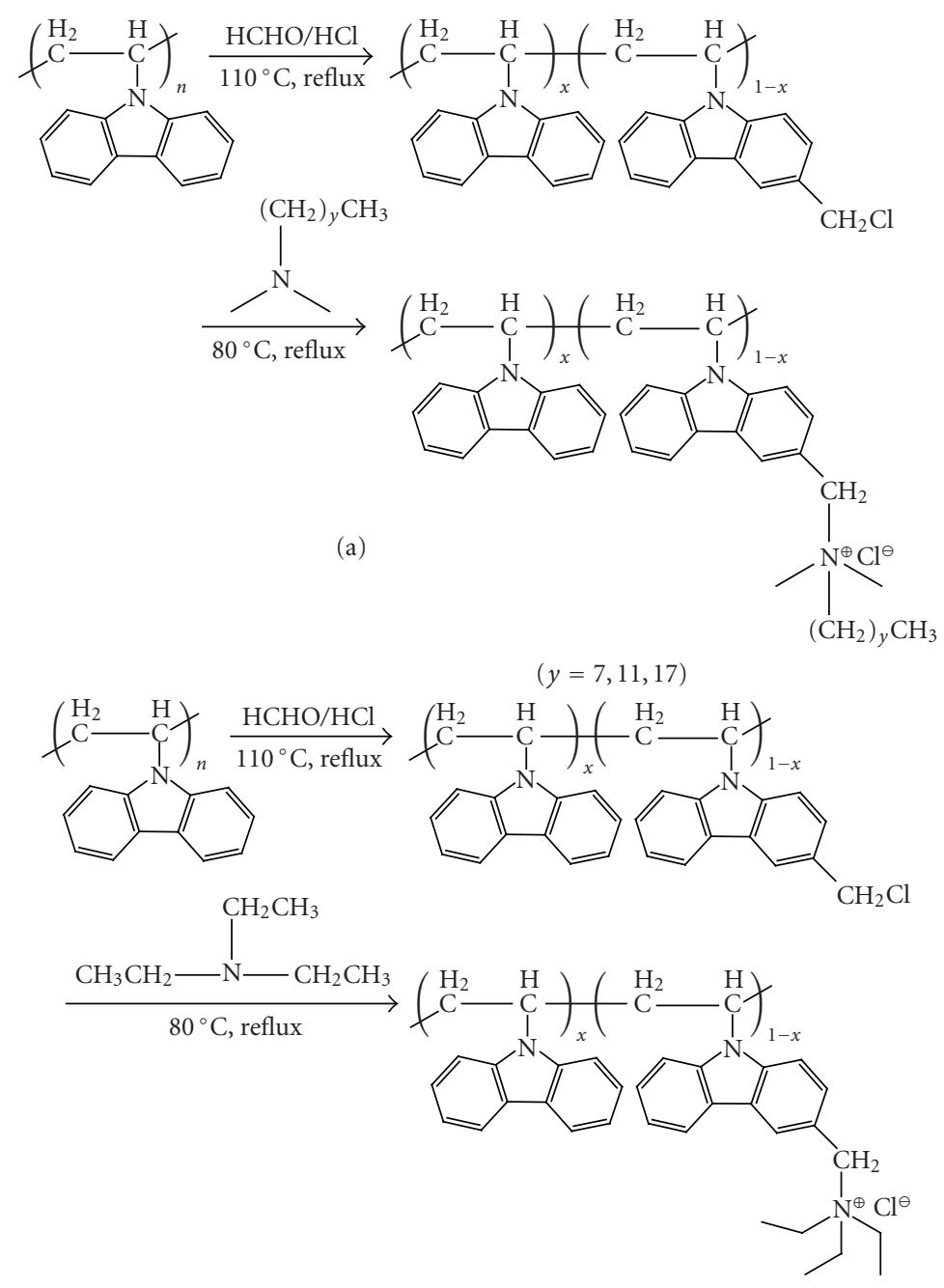

(b)

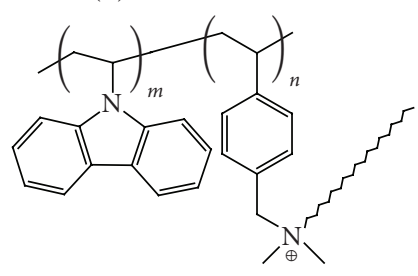

(c)

Scheme 1: (a), (b) Synthesis route of PVK-N ${ }^{+}$polymers. (c) The structure of CPVKOVDAC.

previous studies, the novel polymer had several advantages. It was (1) simple to characterize and had (2) better property of carrier transport. (3) The length of alkyl chains could be changed easily, which allowed us to investigate the effect of alkyl chain length on the PL properties of NCs. (4) The quantum yield of NCs had been improved by $50 \%$ due to the shorter distance between carbazole groups and NCs. The results of interaction between polymers and CdTe NCs indicated that the CdTe NC-PVK-based composites would possess good luminescent or photovoltaic properties just by varying the content of CdTe NCs in the composites.

\section{EXPERIMENTAL}

\subsection{Materials}

Poly(9-vinylcarbazole) (Mw 90000$)$ and tellurium powder (-200 mesh, 99.8\%) were purchased from Sigma-Aldrich Chemical Corporation (Mo, USA). 3-Mercaptopropionic acid (MPA), thioglycolic acid (TGA), N, N-dimethyl octadecylamine, N, N-dimethyl dodecyl amine, N, N-dimethyl octyl amine, and 4-vinylbenzyl chloride were purchased from Acros Organics (UK). $\mathrm{CdCl}_{2}(99+\%), \mathrm{NaBH}_{4}$ (99\%), and 
triethylamine were commercially available products. All of the solvents had analytical grade and were used as received.

\subsection{Synthesis of decorated PVK polymer}

Concentrated hydrochloric acid $(2.3 \mathrm{~mL}, 36.7 \%)$ and aqueous formaldehyde $(1.25 \mathrm{~mL}, 30 \%)$ were added to a stirred solution $\left(0-5^{\circ} \mathrm{C}\right)$ of PVK $(1 \mathrm{~g})$ in 1 , 4 -dioxane $(100 \mathrm{~mL})$. The reaction mixture was allowed to warm to room temperature and was stirred for 1 hour. The reaction mixture was then heated to reflux for 4 hours. Cooling back to $80^{\circ} \mathrm{C}$, tertiary amines were added to the reactant mixture and reacted for 4 hours. The mixtures were poured into a large amount of methanol to remove the unreacted small molecules, and the polymers were obtained by centrifugation. The polymer was dissolved in chloroform and precipitated in methanol several times to achieve pure decorated PVK polymer. Then, the product was kept at $40^{\circ} \mathrm{C}$ in vacuum overnight (FTIR (KBr): 2923, 2851 ( $v, \mathrm{C}-\mathrm{H}$ stretch vibrations in octadecyl), 1624, 1595 (two aromatic vibrations in carbazole moieties), 1482, 1449 (vibrations in five-membered ring of carbazole), 1325, 1227 ( $v, \mathrm{C}-\mathrm{N}$ stretch vibrations)).

\subsection{Preparation of CdTe NC-PVK-based polymer composites}

A series of aqueous solutions of CdTe nanocrystals capped with MPA or TGA was prepared according to previous reports [11]. The polymer was dissolved in chloroform at some concentration. The polymer solution was added to an aqueous CdTe solution ( $0.00125 \mathrm{~N}$ according to $\mathrm{Cd}^{2+}$ ) with vigorous stirring. The organic phase was then separated to yield the CdTe NC-composite solution. The chloroform solvent was removed under reduced pressure and the resulting composite solids were kept in vacuum overnight. The composite solid can be dissolved again in chloroform for characterization.

\subsection{Characterizations}

FTIR spectra were recorded from a $\mathrm{KBr}$ window on a Nicolet AVATAR 360 FTIR spectrophotometer. UV-Vis spectra were acquired using a Shimadzu 3100 UV-Vis spectrophotometer. Fluorescence experiments were performed on a Shimadzu RF-5301 PC spectrofluorimeter. NETZSCH STA 449C thermogravimetric analyzer (TGA) with a heating rate of $10^{\circ} \mathrm{C} / \mathrm{min}$, up to $800^{\circ} \mathrm{C}$, was used for the thermal degradation of the polymers under nitrogen. Element analysis was performed on Elementar Analysensysteme GmbH VarioEL. The quantum yields (QYs) of CdTe NC-polymer composites were determined using quinine sulfate $\left(10^{-5} \mathrm{M}\right.$ in $0.5 \mathrm{~mol} / \mathrm{L}$ $\left.\mathrm{H}_{2} \mathrm{SO}_{4}\right)$ as reference. The absorbance at the excitation wavelength was below 0.1 in order to avoid any significant reabsorption.

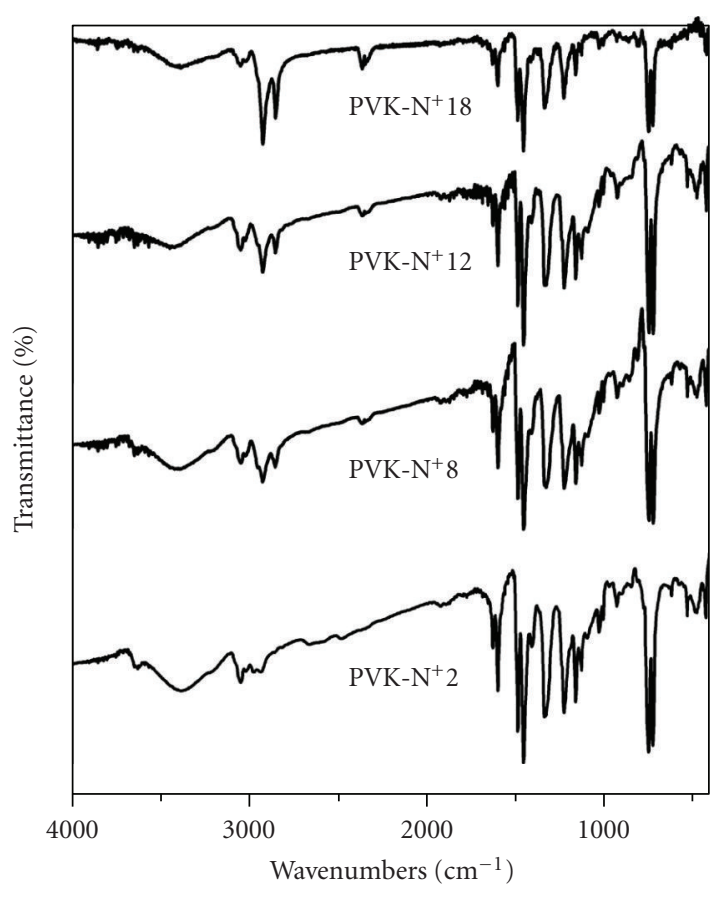

FIGURE 1: FTIR spectra of PVK-N ${ }^{+}$polymers.

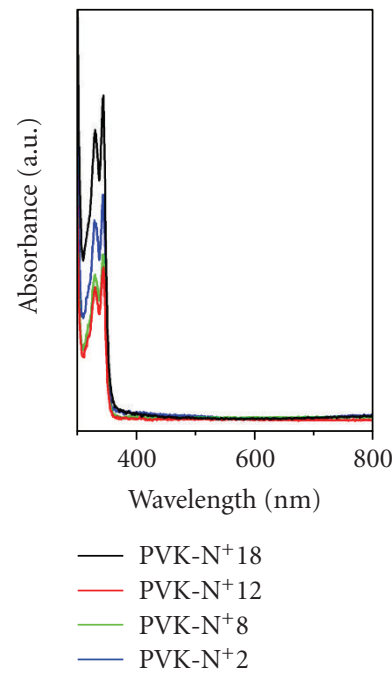

(a)

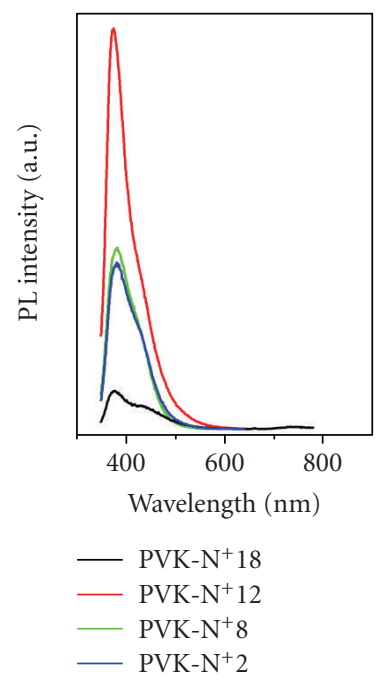

(b)
FIgUre 2: (a) UV-Vis absorption spectra and (b) PL spectra excited at $325 \mathrm{~nm}$ of the $\mathrm{PVK}-\mathrm{N}^{+}$polymers in chloroform.

\section{RESULTS AND DISCUSSION}

\subsection{Synthesis and properties of PVK-based polymer}

PVKs have been considered as potential polymer hosts for high-efficiency blue light-emitting diodes, and they found applications in photovoltaics, photoconductive materials, electroluminescence (EL) devices, photorefractive materials, nonlinear optical devices, and so forth [12-16]. It has been reported that chloromethylated PVK was functionalized with 
TABLe 1: Basic information of the PVK-N ${ }^{+}$polymers.

\begin{tabular}{|c|c|c|c|}
\hline $\begin{array}{l}\text { Abbreviations of } \\
\text { the polymers }\end{array}$ & $\begin{array}{l}\text { Tertiary amine used in the second } \\
\text { step of the reaction }\end{array}$ & $\begin{array}{l}\text { Numbers of quaternary amine deco- } \\
\text { rated on the polymer chain per } 100 \\
\text { carbazole moieties }\end{array}$ & $\begin{array}{l}\text { Decomposition temperature } \\
\left({ }^{\circ} \mathrm{C}\right)(5 \% \text { weight loss })\end{array}$ \\
\hline $\mathrm{PVK}^{-\mathrm{N}^{+}} 18$ & $\mathrm{~N}, \mathrm{~N}$-dimethyl octadecylamine & 25 & 215 \\
\hline PVK-N ${ }^{+} 12$ & $\mathrm{~N}, \mathrm{~N}$-dimethyl dodecylamine & 18 & 201 \\
\hline $\mathrm{PVK}-\mathrm{N}^{+} 8$ & $\mathrm{~N}, \mathrm{~N}$-dimethyl octylamine & 21 & 211 \\
\hline PVK-N ${ }^{+} 2$ & Triethylamine & 15 & 209 \\
\hline
\end{tabular}

Disperse Red 1 (DR-1) by Williamson ether synthesis [17]. Because of the higher reactivity of the carbazole ring toward electrophilic substitution, a serious cross-linking reaction occurred in preparation of chloromethylated PVK using chloromethyl methyl ether and zinc chloride, leading to the low content of chloromethyl in the soluble part of chloromethylated PVK. Consequently, further chromophoric functionalization was not easy to complete. In our studies, it was also found that the molecular weight, temperature of reaction, time of reflux, and so forth had an important influence on the resulted polymers. The formation of crosslinking polymers would occur if the experiment conditions were not well controlled. Scheme 1 gives the synthesis route of PVK-based polymer and Table 1 shows the basic information of the polymers. For a given molecular weight of PVK, for example, $\mathrm{Mw}=90000$, the concentration of PVK in 1, 4-dioxane should be controlled below $10 \mathrm{mg} / \mathrm{mL}$. The temperature of reaction should be controlled at $80-110^{\circ} \mathrm{C}$. Temperature below this will result in low degree of chloromethylation and low yield of PVK-based polymer at last. The reflux time must be shorter than 4 hours in the first step of the reaction in order to avoid the formation of cross-linking polymers. In the second step of the reaction, the tertiary amine probably reacts with surplus hydrochloric acid in the first step to form small quaternary amine molecules. Therefore, we added excessive tertiary amine to insure that there was enough tertiary amine to react with the chloromethylated PVK polymers. The PVK-based polymer was dissolved in chloroform and precipitated in methanol to delete the small quaternary amine molecules. Figure 1 shows the FTIR spectra of the PVK-based polymers. We can clearly observe strong $\mathrm{C}-\mathrm{H}$ stretch vibration peaks at 2923 and $2851 \mathrm{~cm}^{-1}$, which resulted from alkyl in the amine moieties. The peaks at 1624 and $1595 \mathrm{~cm}^{-1}$ are attributed to the two aromatic vibrations in carbazole moieties. The peaks at 1482 and $1449 \mathrm{~cm}^{-1}$ resulted from the five-membered ring of carbazole. The peaks at 1325 and $1227 \mathrm{~cm}^{-1}$ are attributed to $\mathrm{C}-\mathrm{N}$ stretch vibration. These characteristic peaks have verified the formation of PVK-based polymers. The numbers of quaternary amine decorated on the polymer chain are also listed in Table 1, which are estimated from element analysis (see Table 2). The decomposition temperature of the polymers was about $200^{\circ} \mathrm{C}$, which could satisfy the requirement of fabricating device using spin-coating method.

Figure 2 shows the UV-Vis absorption and PL spectra of the polymers. The electron transition within carbazole moieties leads to the peaks at 343 and $330 \mathrm{~nm}$ of absorption spectra. The peaks at $365 \mathrm{~nm}$ of PL spectrum are attributed to
TABLE 2: Element analysis of the PVK- $\mathrm{N}^{+}$polymers.

\begin{tabular}{lcccc}
\hline & $\mathrm{C}(\%)$ & $\mathrm{H}(\%)$ & $\mathrm{N}(\%)$ & $\mathrm{Cl}(\%)$ \\
\hline PVK-N $^{+} 18$ & 82.415 & 7.415 & 6.291 & 1.640 \\
PVK-N $^{+} 12$ & 83.310 & 6.379 & 6.936 & 1.000 \\
PVK-N $^{+} 8$ & 83.145 & 6.352 & 7.074 & 1.600 \\
PVK-N $^{+} 2$ & 83.260 & 5.760 & 7.201 & 1.700 \\
\hline
\end{tabular}

the high-energy excimer radiation of carbazole moieties. The UV-Vis and PL spectra indicate that the resultant polymers retain the photophysical properties of PVK.

\subsection{Composite of semiconductor Nanocrystals and PVK-N+}

The PVK-N ${ }^{+}$polymers are readily soluble in common organic solvents such as chloroform, dichloromethane, N, Ndimethylformamide, and so forth. Different CdTe NCs as prepared in aqueous medium were directly transferred to the organic phase using the PVK- ${ }^{+} 18$ polymers to achieve composites 1-5, respectively (see Table 3 ). In this process, the electrostatic interaction between negatively charged CdTe NCs and positively charged moieties in polymers was the driving force for the formation of composite. Figures 3(a) and 3(b) show the UV-Vis absorption and PL spectra of the resultant composites $\mathbf{1 - 5}$ in chloroform. Absorption and PL spectra at different wavelengths correspond to distinct CdTe NCs, which confirms the presence of NCs in the polymer and the successful transfer of NCs from the aqueous phase to the organic phase. Furthermore, the full width at half maximum (FWHM) of PL peaks of the composite is similar to that of the parent NCs as prepared in aqueous medium, which indicates that the excellent properties of CdTe NCs were retained after they were capped with polymers. A clear solution of NCs in organic solvent strongly indicates a homogenous distribution of NCs in the composites (see Figure 3(c)). The QYs of CdTe NCs in the composites $\mathbf{1 - 5}$ are $0.612,0.476$, $0.168,0.368$, and 0.146, respectively. For composites 1, 2, 3, and 5, the QYs are higher than their parent NC aqueous solutions. When we used polymer CPVKOVDAC to obtain NC composites, the QYs of CdTe NCs in the composites were always lower than parent NC aqueous solutions $[9,10]$. The improvement of QY in this study is believed to be brought by the shorter distance from carbazole moieties to CdTe NCs in PVK-N ${ }^{+}$system, which facilitates the Förster resonant energy transfer (FRET) between them. 
TABLE 3: Component and quantum yields (QYs) of the composites.

\begin{tabular}{llcc}
\hline $\begin{array}{l}\text { Abbreviations of } \\
\text { the composites }\end{array}$ & CdTe NCs used & $\begin{array}{l}\text { QYs }^{(\mathrm{b})} \text { of CdTe NCs } \\
\text { in aqueous solution }\end{array}$ & $\begin{array}{l}\text { QYs of CdTe NCs } \\
\text { in the composites }\end{array}$ \\
\hline Composite $\mathbf{1}$ & TGA $(530 \mathrm{~nm})^{(\mathrm{a})}$ & 0.400 & 0.612 \\
Composite $\mathbf{2}$ & TGA $(540 \mathrm{~nm})$ & 0.462 & 0.476 \\
Composite $\mathbf{3}$ & MPA $(490 \mathrm{~nm})$ & 0.141 & 0.168 \\
Composite $\mathbf{4}$ & MPA $(540 \mathrm{~nm})$ & 0.395 & 0.368 \\
Composite $\mathbf{5}$ & MPA $(600 \mathrm{~nm})$ & 0.108 & 0.146 \\
\hline
\end{tabular}

(a) Reference to the CdTe NCs capping with TGA ligand and absorption of exciton at $530 \mathrm{~nm}$. ${ }^{\text {(b) }}$ Using quinine sulfate $\left(10^{-5}\right)$ as PL reference.

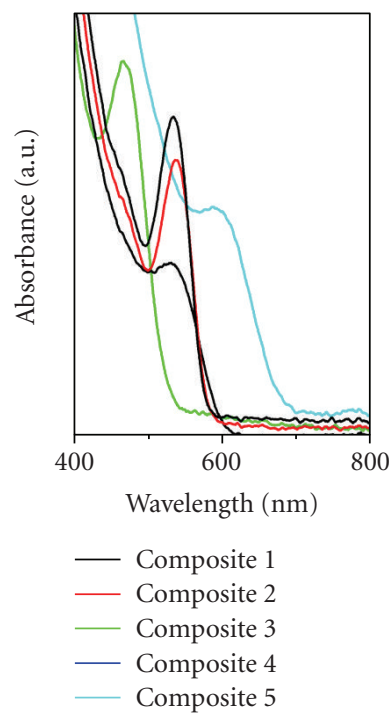

(a)

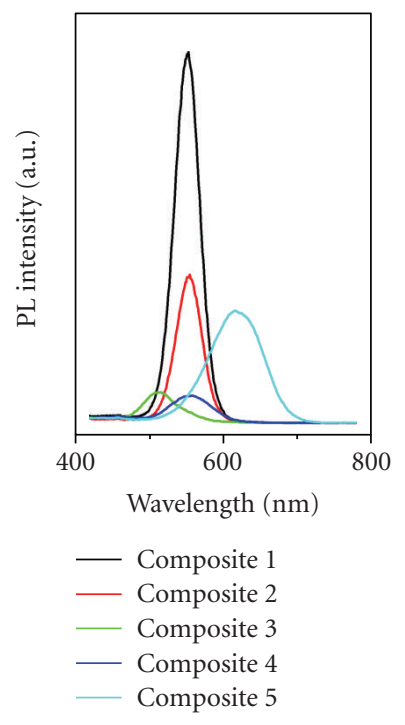

(b)

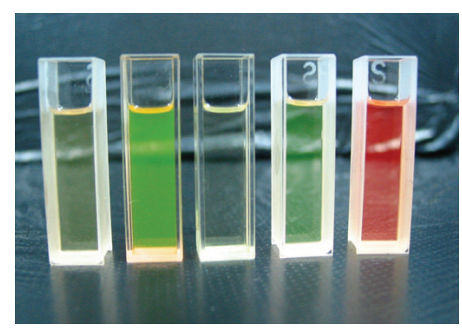

(c)

Figure 3: (a) UV-Vis absorption and (b) PL spectra excited at $400 \mathrm{~nm}$ of CdTe NC-polymer composites 1-5 in chloroform containing different CdTe NCs. (c) Photographs of composites $\mathbf{1 - 5}$ in chloroform.

\subsection{Interaction between CdTe NCs and PVK-N ${ }^{+}$}

Several methods have been developed to obtain the composites such as directly mixing the NCs into the polymers, in situ synthesis of NCs in polymer matrix, layer-by-layer assembly to achieve NC-polymer composite, and so forth [1824]. Although some composites have been obtained using these methods, new problems also confronted chemists, such as aggregation of NCs in polymer matrix and PL quenching due to the different kinds of interaction between polymers and NCs. In our system, the process of composition of poly-

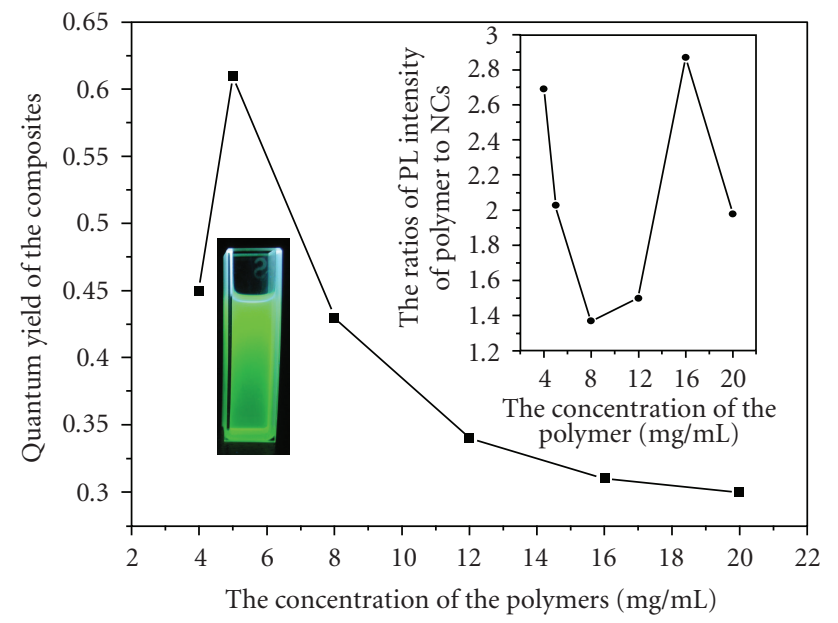

FIgURE 4: The curves of QYs versus concentration of polymer. Inset is the photograph of the composites with QY of 0.612, and the curves of the ratios of PL intensity of polymer to NCs versus concentration of polymer.

mer and CdTe NCs overcomes the major problems associated with the miscibility of NCs with polymer matrix and PL quenching of NCs in the polymer. However, we did find that the photophysical properties of NCs were influenced by the electrostatic interaction between polymers and NCs. Figure 4 shows that the QYs of the composites increase with increasing polymer concentration when the concentration of the polymer is below $5 \mathrm{mg} / \mathrm{mL}$ (the contents of CdTe were kept constant and the CdTe NCs were completely transferred from aqueous phase to organic phase). We conclude that the interaction between carbazole and CdTe NCs is mainly FRET whose energy is transferred by the excited carbazole moieties to CdTe NCs via dipole-dipole interaction at such concentrations. The highest QY was 0.612 when the concentration of the polymer was equal to $5 \mathrm{mg} / \mathrm{mL}$. Inset of Figure 4 is the photo of the composite with the highest QY under UV illumination. The QY of CdTe NCs decreases with increasing polymer concentration when the concentration of the polymer is higher than $5 \mathrm{mg} / \mathrm{mL}$. We suppose that electron transfer from carbazole moieties and CdTe NCs occurred, which quenched the PL of the resultant composites. For the PVK- ${ }^{+}$polymer, the PL intensity increases with increasing polymer concentration from $5 \mathrm{mg} / \mathrm{mL}$ to $20 \mathrm{mg} / \mathrm{mL}$. However, from the inset curve of Figure 4, it can be observed that 


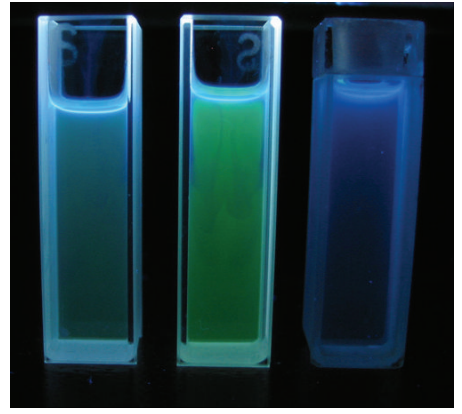

(a)

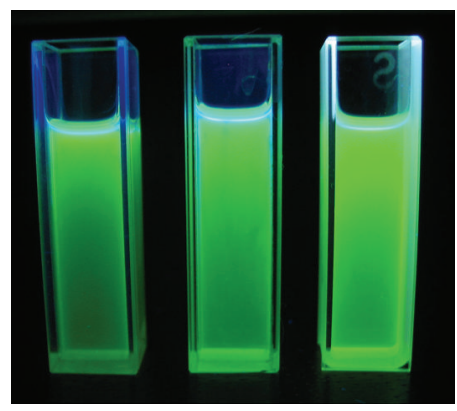

(b)

FIGURE 5: The photographs of (a) the composites with low-content CdTe NCs (the QYs of the NCs in the composite were 0.043, 0.107, and 0.061 from left to right) and (b) high-content CdTe NCs (the QYs of the NCs in the composite were $0.489,0.571$, and 0.612 from left to right). The photographs of (a) and (b) were taken under the same condition. The composites with low content of CdTe NCs refer to the composites obtained via $10 \mathrm{~mL}(5 \mathrm{mg} / \mathrm{mL})$ PVK-N ${ }^{+}$polymer solution adding 10, 20, $40 \mathrm{~mL}$ CdTe NC aqueous solutions, while the composites with high content of CdTe NCs refer to the composites obtained via $10 \mathrm{~mL}(5 \mathrm{mg} / \mathrm{mL})$ PVK-N ${ }^{+}$polymer solution adding 60, 80, 100 mL CdTe NC aqueous solutions.

the ratios of PL intensity of polymer to NCs decrease first and then increase when the polymer concentration increases continuously. These results indicate that the most efficient FRET occurred at the point at which the concentration of the polymer was about $8 \mathrm{mg} / \mathrm{mL}$.

The tendency of PL changes of the composites became much more obvious when the concentration of the polymer was set constant, namely, $5 \mathrm{mg} / \mathrm{mL}$, and the content of $\mathrm{NCs}$ was varied at some range. In CdTe $\mathrm{NC}$ aqueous solution, the QYs of the NCs increase with decreasing NC concentration because the self-quenching occurs at higher concentration. In the composite system, however, when the concentration of the polymer was about $5 \mathrm{mg} / \mathrm{mL}$, the QYs of the NCs were higher in the composites with high content of CdTe NCs than those of the composites with low content of CdTe NCs. Figure 5 gives the photos of the composites with different contents of CdTe NCs under UV illumination. It can be clearly observed that the composites with low content of CdTe NCs show weak PL intensity (see Figure 5(a); the QYs of the NCs in the composite are 0.043, 0.107, 0.061 from left to right) while the ones with high content of CdTe NCs show strong PL intensity (see Figure 5(b); the QYs of the NCs in the composite are $0.489,0.571,0.612$ from left to right). This phenomenon verifies the conclusion we have drawn above. We have ever systematically studied in our recent work [25] the mechanism of interaction between carbazole moieties and CdTe NCs. It is believed that the excited carbazole moieties could form carbazole anions and carbazole cations via resonance with carbazole moieties at the ground state under UV illumination. The carbazole anions migrated to the positively charged surface of CdTe NC-polymer composite and then shortened the distance between carbazole moieties and CdTe NCs. Carbazole anions donated their electrons to CdTe $\mathrm{NCs}$, and then the donated electrons recombined with the holes in CdTe NCs. The result of electron transfer was that holes were confined to polymers while electrons were limited within CdTe NCs. The more the polymer was, the more positive net charges and carbazole moieties were. Therefore, the electron transfer became efficient with decreasing CdTe NCs.

\section{CONCLUSION}

In summary, we prepared a novel kind of PVK-based amphiphilic polymer with luminescent properties. CdTe NCs were directly transferred to the organic phase using the PVKbased polymers to achieve composites. The quantum yield of NCs had been improved by $50 \%$ compared with their parent aqueous solution due to the shorter distance from carbazole groups to NCs which facilitated the FRET between them. More efficient electron transfer at the interface of NCs and polymers was confirmed for composites by decreasing NC content. The results indicated that the composites could found applications in LEDs or photovoltaic devices.

\section{ACKNOWLEDGMENTS}

This work is financially supported by the Special Funds for Major State Basic Research Projects (Grant no. 2002CB613401), the National Natural Science Foundation of China (Grant no. 20534040), the Program for Changjiang Scholars and Innovative Research Team in University (Grant no. IRT0422), the Program of Introducing Talents of Discipline to Universities (Grant no. B06009), and Science Foundation for Young Teachers of Northeast Normal University (Grant no. 20070306).

\section{REFERENCES}

[1] F. Huang, L. Hou, H. Wu, et al., "High-efficiency, environment-friendly electroluminescent polymers with stable high work function metal as a cathode: green- and yellowemitting conjugated polyfluorene polyelectrolytes and their neutral precursors," Journal of the American Chemical Society, vol. 126, no. 31, pp. 9845-9853, 2004.

[2] J. L. Casson, D. W. McBranch, J. M. Robinson, et al., "Reversal of interfacial dipole orientation in polyelectrolyte superlattices due to polycationic layers," Journal of Physical Chemistry $B$, vol. 104, no. 50, pp. 11996-12001, 2000.

[3] K. Doré, S. Dubus, H.-A. Ho, et al., "Fluorescent polymeric transducer for the rapid, simple, and specific detection of 
nucleic acids at the zeptomole level," Journal of the American Chemical Society, vol. 126, no. 13, pp. 4240-4244, 2004.

[4] D. T. McQuade, A. E. Pullen, and T. M. Swager, "Conjugated polymer-based chemical sensors," Chemical Reviews, vol. 100, no. 7, pp. 2537-2574, 2000.

[5] M. Bruchez Jr., M. Moronne, P. Gin, S. Weiss, and A. P. Alivisatos, "Semiconductor nanocrystals as fluorescent biological labels," Science, vol. 281, no. 5385, pp. 2013-2016, 1998.

[6] W. C.W. Chan and S. Nie, "Quantum dot bioconjugates for ultrasensitive nonisotopic detection," Science, vol. 281, no. 5385, pp. 2016-2018, 1998.

[7] A. Schroedter, H. Weller, R. Eritja, W. E. Ford, and J. M. Wessels, "Biofunctionalization of silica-coated CdTe and gold nanocrystals," Nano Letters, vol. 2, no. 12, pp. 1363-1367, 2002.

[8] S. Wang, N. Mamedova, N. A. Kotov, W. Chen, and J. Studer, "Antigen/antibody immunocomplex from CdTe nanoparticle bioconjugates," Nano Letters, vol. 2, no. 8, pp. 817-822, 2002.

[9] H. Sun, J. Zhang, H. Zhang, et al., "Preparation of carbazolecontaining amphiphilic copolymers: an efficient method for the incorporation of functional nanocrystals," Macromolecular Materials and Engineering, vol. 291, no. 8, pp. 929-936, 2006.

[10] H. Sun, J. Zhang, H. Zhang, et al., "Pure white-light emission of nanocrystal-polymer composites," ChemPhysChem, vol. 7, no. 12, pp. 2492-2496, 2006.

[11] H. Zhang, Z. Zhou, B. Yang, and M. Gao, "The influence of carboxyl groups on the photoluminescence of mercaptocarboxylic acid-stabilized CdTe nanoparticles," Journal of Physical Chemistry B, vol. 107, no. 1, pp. 8-13, 2003.

[12] A. van Dijken, J. J. A. M. Bastiaansen, N. M. M. Kiggen, et al., "Carbazole compounds as host materials for triplet emitters in organic light-emitting diodes: polymer hosts for high-efficiency light-emitting diodes," Journal of the American Chemical Society, vol. 126, no. 24, pp. 7718-7727, 2004.

[13] J. L. Kim, J. K. Kim, H. N. Cho, D. Y. Kim, C. Y. Kim, and S. I. Hong, "New polyquinoline copolymers: synthesis, optical, luminescent, and hole-blocking/electron-transporting properties," Macromolecules, vol. 33, no. 16, pp. 5880-5885, 2000.

[14] E. A. Plummer, A. van Dijken, H. W. Hofstraat, L. De Cola, and K. Brunner, "Electrophosphorescent devices based on cationic complexes: control of switch-on voltage and efficiency through modification of charge injection and charge transport," Advanced Functional Materials, vol. 15, no. 2, pp. 281289, 2005.

[15] X. Gong, J. C. Ostrowski, D. Moses, G. C. Bazan, and A. J. Heeger, "Electrophosphorescence from a polymer guesthost system with an iridium complex as guest: förster energy transfer and charge trapping," Advanced Functional Materials, vol. 13, no. 6, pp. 439-444, 2003.

[16] J. G. Winiarz, L. Zhang, M. Lal, C. S. Friend, and P. N. Prasad, "Photogeneration, charge transport, and photoconductivity of a novel PVK/CdS-nanocrystal polymer composite," Chemical Physics, vol. 245, no. 1-3, pp. 417-428, 1999.

[17] J. Luo, J. Qin, H. Kang, and C. Ye, "A postfunctionalization strategy to develop PVK-based nonlinear optical polymers with a high density of chromophores and improved processibility," Chemistry of Materials, vol. 13, no. 3, pp. 927-931, 2001.

[18] B. O. Dabbousi, M. G. Bawendi, O. Onitsuka, and M. F. Rubner, "Electroluminescence from CdSe quantum-dot/polymer composites," Applied Physics Letters, vol. 66, no. 11, pp. 13161318, 1995.
[19] S. Wang, S. Yang, C. Yang, Z. Li, J. Wang, and W. Ge, "Poly(Nvinylcarbazole) (PVK) photoconductivity enhancement induced by doping with CdS nanocrystals through chemical hybridization," Journal of Physical Chemistry B, vol. 104, no. 50, pp. 11853-11858, 2000.

[20] M. Gao, C. Lesser, S. Kirstein, E. Möhwald, A. L. Rogach, and H. Weller, "Electroluminescence of different colors from polycation/CdTe nanocrystal self-assembled films," Journal of Applied Physics, vol. 87, no. 5, pp. 2297-2302, 2000.

[21] M. Gao, J. Sun, E. Dulkeith, N. Gaponik, U. Lemmer, and J. Feldmann, "Lateral patterning of CdTe nanocrystal films by the electric field directed layer-by-layer assembly method," Langmuir, vol. 18, no. 10, pp. 4098-4102, 2002.

[22] D. Wang, A. L. Rogach, and F. Caruso, "Semiconductor quantum dot-labeled microsphere bioconjugates prepared by stepwise self-assembly," Nano Letters, vol. 2, no. 8, pp. 857-861, 2002.

[23] T. Franzl, T. A. Klar, S. Schietinger, A. L. Rogach, and J. Feldmann, "Exciton recycling in graded gap nanocrystal structures," Nano Letters, vol. 4, no. 9, pp. 1599-1603, 2004.

[24] T. Franzl, A. Shavel, A. L. Rogach, et al., "High-rate rnidirectional energy transfer in directly assembled CdTe nanocrysta bilayers," Small, vol. 1, no. 4, pp. 392-395, 2005.

[25] H. Sun, H. Zhang, J. Zhang, et al., "Effect of electrostatic interaction on the photophysical properties of the composites of CdTe nanocrystals and carbazole-containing polymers," in preparation. 

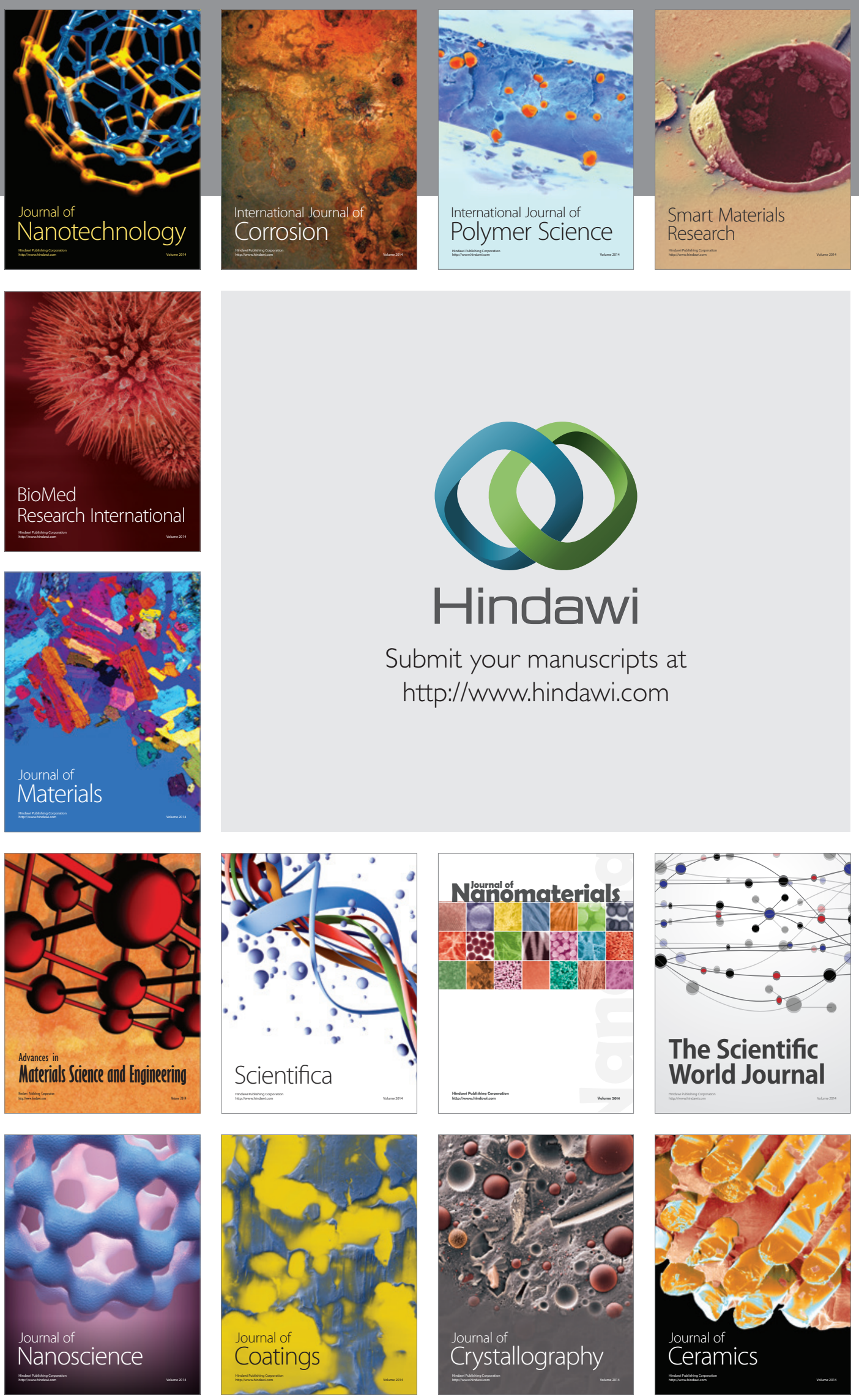

The Scientific World Journal

Submit your manuscripts at

http://www.hindawi.com

\section{World Journal}

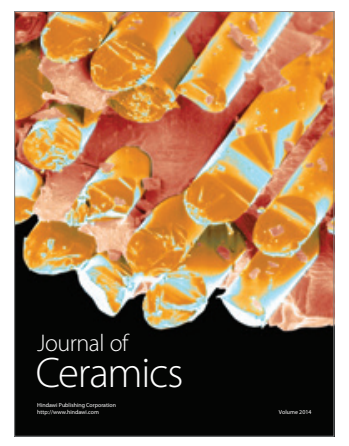

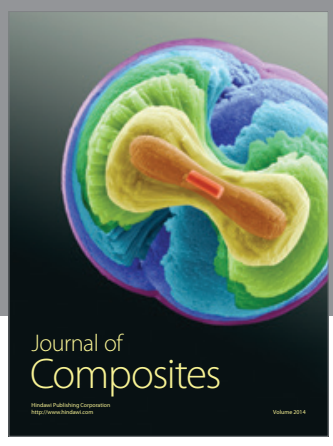
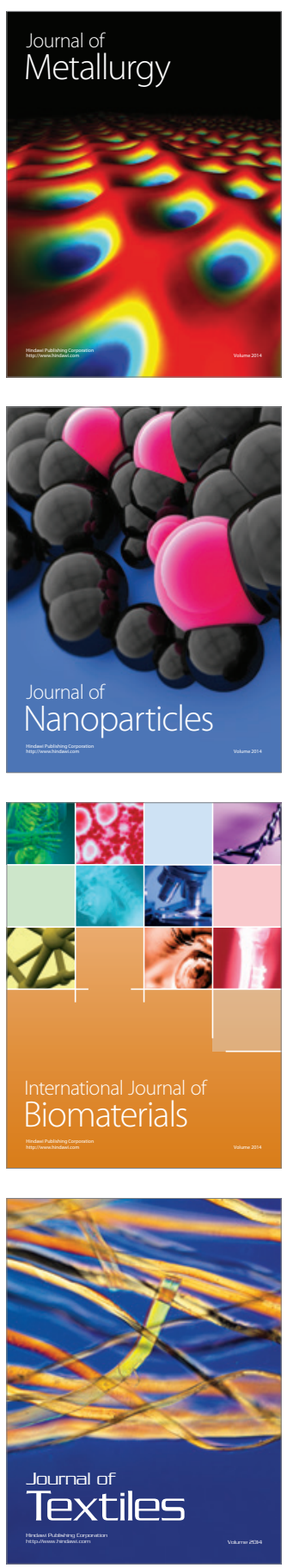